\title{
Real-time Heart Pulse Monitoring Technique Using Wireless Sensor Network and Mobile Application
}

\author{
Nabeel Salih Ali ${ }^{1}$, Zaid Abdi Alkaream Alyasseri ${ }^{2}$, Abdulhussein Abdulmohson ${ }^{3}$ \\ ${ }^{1}$ ITRDC Center, University of Kufa, Iraq \\ ${ }^{2}$ Faculty of Engineering, University of Kufa, Iraq \\ ${ }^{3}$ Faculty of Education, University of Kufa, Iraq
}

\section{Article Info}

Article history:

Received Apr 9, 2018

Revised Mei 28, 2018

Accepted Jul 16, 2018

\section{Keyword:}

Arduino

Body Sensor Network Systems

Healthcare

Heart Pulse

Real-Time

Smartphone

Wireless Sensor Network

\begin{abstract}
Wireless Sensor Networks (WSNs) for healthcare have emerged in the recent years. Wireless technology has been developed and used widely for different medical fields. This technology provides healthcare services for patients, especially who suffer from chronic diseases. Services such as catering continuous medical monitoring and get rid of disturbance caused by the sensor of instruments. Sensors are connected to a patient by wires and become bed-bound that less from the mobility of the patient. In this paper, proposed a real-time heart pulse monitoring system via conducted an electronic circuit architecture to measure Heart Pulse (HP) for patients and display heart pulse measuring via smartphone and computer over the network in real-time settings. In HP measuring application standpoint, using sensor technology to observe heart pulse by bringing the fingerprint to the sensor via used Arduino microcontroller with Ethernet shield to connect heart pulse circuit to the internet and send results to the web server and receive it anywhere. The proposed system provided the usability by the user (userfriendly) not only by the specialist. Also, it offered speed andresults accuracy, the highest availability with the user on an ongoing basis, and few cost.
\end{abstract}

Copyright $\odot 2018$ Institute of Advanced Engineering and Science. All rights reserved.

\section{Corresponding Author:}

Nabeel Salih Ali,

Information Technology Research and Development Center (ITRDC), University of Kufa,

Kufa Street, Main Campus, University of Kufa, Najaf Governorate, Iraq.

Email: Nabeel@uokufa.edu.iq

\section{INTRODUCTION}

The global elderly population is growing, and the general population is aging, therefore, monitoring systems for hospital healthcare are necessary to continually monitor and track the physiological parameters of the patient [1-3]. Modern applications have been presented via wireless technologies to bring forth exciting possibilities in the medical markets [4]-[5]. Whilst, several portable devices are produced to perform monitoring and tracking tasks to the physiological parameters for the patient. These materials include heart rate monitors, pulse ox meters, and blood pressure [6]-[8]. Additionally, the patient can be attached to these instruments via wired and become bed-bound in moving sequentially As well as discrete continuously can appear in the monitoring devices via they have to be disconnected and then reconnected later [9]-[10]. Today, wireless technology has been eliminated all of the time-consuming jobs as well; the patients could be independent of instrumentation and bed [11]-[13].

Wireless Sensor Network (WSN) is one of the standard direction in information communication and technology (ICT) research areas that developed on various technologies such as sensor technology, microelectromechanical technology and wireless communication technology [14]-[16]. Consequently, several wireless systems have been rapidly increasing primarily for medical applications in the recent years [17]. 
With using Body Sensor Network (BSN) systems, the life quality for people has been improved via providing different healthcare services such as medical monitoring, memory enhancement, and medical data access communication with the healthcare provider in emergency situations through the Short Message Service (SMS) or General Packet Radio Services (GPRS) [18]-[20]. Healthcare services can be benefited for the patient and their families by providing a remotely gain and monitor physiological signals without the necessity of interruption the healthy life of patient [21].

Smart healthcare possibly vision is produced by several studies in all computer, networking and medical care domains. Researchers have increasingly become interested in improving technical solutions that address problems in healthcare delivery [22]. However, accurately predicting the future of any healthcare domain is a complicated task. Hence, delivery of healthcare services to members of the global aging population poses significant challenges. Moreover, delivery of these services is affected by different situations that require cost cutting for healthcare services [23].

Aminian et al, 2013, proposed health monitoring system to monitor the patient physical parameters for pregnant woman parameters mainly; These parameters are blood pressure and heart rate of the woman and heart rate and movements of fetal by the wireless sensor network; In the proposed system, sent data with short range of frequency by using relay and wireless relay with minimum energy consumption provide quality whilst, this system has some problems, one of these issues the coordinator node that works with battery while the patient forgot to recharge the battery that causes dangerous for the patient life [24]. Likewise, González et al (2014), conducted a new alternative to measure heart rate and body temperature for patient care in real-time settings via combined between Wireless Sensor Network (WSN) and Mobile Augmented Reality (MAR); The discussed work can be extended to involve some improvements such as database implementation with the cloud and more manufacturing specifications for sensors [25].

As alongside, Triantafyllidis (2014), proposed a smartphone healthcare system to measure physiological parameters of the patient by using portable/wearable sensors and monitor the condition of a patient and configured monitoring plans for the health professionals in an individualized manner [26]. Almadani et al (2015), conducted an E-Ambulance framework of smart health monitoring system of patients for the remote professional medical model; The presented method provided a paramedic staff with automatic responses to warnings and suggestions inside an ambulance. But, it lacks to use diverse medical systems over different wireless technologies like ZigBee, WI-Fi, and Bluetooth technologies [27]. On the other hands, a real-time monitoring system for various health parameters of a patient was implemented by Mahgoub and Khalifa (2015).

The measured parameters include temperature and Oxygen that are calculated and transmitted by a computer based on a remote client; There are some limitations of their results such as affecting noise which obtained from sensors, permanent damage to the Light Emitting Diode (LED) sensors, and there is a little bit complicated when calculating the SpO2 [28]. As well, a new model was proposed by Tamura et al. (2015), to improve team-based healthcare for testing and to monitor the physiological parameters of patients which can be used as a rehabilitation training system. The proposed method included a highly efficient database, unobtrusive monitoring, and interventions by health professionals; Whilst, the system required more testing in a home-based healthcare environment [29]. Akshay and Krishna (2016), designed and presented a healthcare improvement system which can be used to providing higher quality healthcare services in the population of Rural India. The proposed system can be easily carried and rapidly measurement with implemented algorithms; Whilst, the system required helping a doctor to detect abnormal activity and to keep tracking of those particular person/ patient [30].

In this paper, design a system application for measuring heart pulse for the patient and displaying the heart pulse via a smartphone and computer over the network in real time environment. The proposed system using a heart pulse sensor, Arduino Uno microcontroller, Ethernet shield for remote healthcare monitoring for the patients. The system includes several steps such as sensing and reading circuit, processing and transferring signals, and finally connecting to the network to present the results. Additionally, the sensing reading will be presented via computer and smartphone-based applications. The rest of the paper structure as follows: Section 2 highlights the methodology of the proposed system that contains: materials and methods description, implementation steps. Explain system testing as well the results of the proposed system and discussions in Section 3. Section 4 presents a comparison of the heart rate monitoring systems that have been done in healthcare services and applications concerning system performance and results. Besides, the last section offers conclusions and future directions which is Section 5.

\section{METHODOLOGY OF THE PROPOSING REAL-TIME SYSTEM}

This section presents and describes the methods of the proposed monitoring system that include system architecture defines in Section 2.1, system implementation visualizes in Section 2.2.

Real-time Heart Pulse Monitoring Technique using Wireless Sensor Network and Mobile ... (Nabeel S.A.) 


\subsection{System Architecture}

This section presents the electronic circuit design that includes software and hardware requirements as well as methods of the proposed system monitoring. The system aims to measure and analyze the Heart Pulse (HP) of patients. The results are stored in a computer-based application which can be accessed remotely as well as via smartphone application based.

Hardware and software components are required to implement the electronic circuit. These elements were selected based on different criteria namely low cost, availability, and ease of programming. The hardware components needed for the implementation such as Arduino UNO board, amplifier (LM368), filter LBF, Ethernet shield V6, sensors (IR Emitter), and Samsung Galaxy s Duos S7562. Also, the required software components are Arduino program V1.6.0, processing program (V 3.0.1), Android V1.6.0, and Android studio. Figure 1 presents the block diagram of the proposed monitoring system.

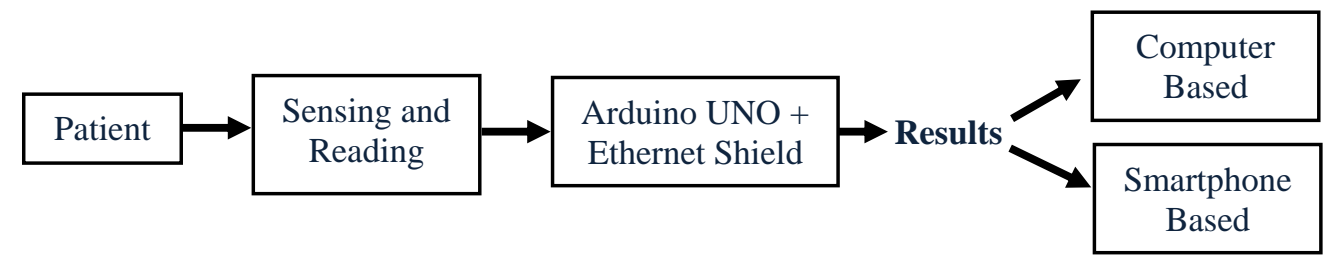

Figure 1. Block diagram for the proposed system architecture

\subsection{System Implementation}

The flow of blood recorded and measured by the number of pulses in one minute whereas heart rate or heart pulse must be measured accurately due to the importance of measuring the accurate heart rate should not be ignored [31]. In this paper, a hybrid scheme between wireless sensor network (WSN) and smartphone application for heart pulse monitoring is proposed. The main purpose of the proposed system is to monitor and check the status of the patient in real-time environment and report the result via computer-based application and smartphone-based application. Figure 2 shows the implementation steps of the proposed system where it involves three stages that are describing in the next subsections respectively (2.2.1, 2.2.2, 2.2.3).

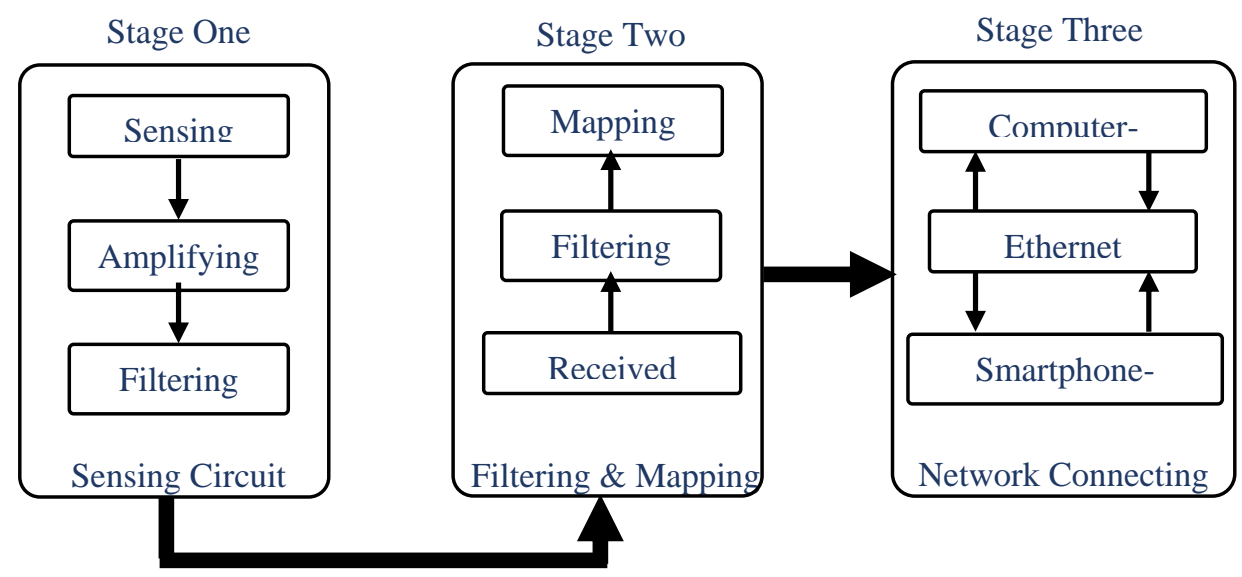

Figure 2. Implementation steps for the proposed monitoring system

In this first stage (sensing circuit stage) the heart pulse will be detected by using the blood racing through veins. Then, the heart pulse will process via amplifying and filtering to be more suitable for the next step. In the second stage (filtering and mapping stage), the heartbeat signal which is received from the previous stage will be filtered again to ensure that it is free of noise. After that, it will be mapping to utilize smartphones and computer devices. The last stage (network connecting) is connecting and display the results of the heart pulse via smartphone or computer devicesconsecutively. 


\subsubsection{Stage One (Sensing Circuit Stage)}

In heart pulse measuring (sensing) standpoint, the system using sensor technology to detect the blood racing through veins via identifying the heart pulse of a patient and counts the pulses for one minute to get the beats per minute. The sensing process is done by bringing the patient's fingerprint to the sensor (emitters and detectors for infrared) which is included two steps that are (IR Emitter) emitting and sensor diodes to detect and measure the pulse that passes light from one side of the finger as well measuring the intensity of the passed light from the other side via using an IR detector see Figure 3 (A). Also, the second process in the sensing circuit stage has used an amplifier (LM386) to amplifying the pulse that measured in the previous procedure. Where, the received signal amplified enough to be detectable by the microcontroller inputs see Figure 3 (B). Whereas the third process in the first stage is done via using filters (LPF). This process using two stages with low pass filters that removed some unwanted signals, which eliminating some frequencies to suppress interfering signals and reduce background noise see Figure 3 (C). Figure 3 include three parts which are (A, B, and C) to present all steps and processes of the sensing circuit stage.

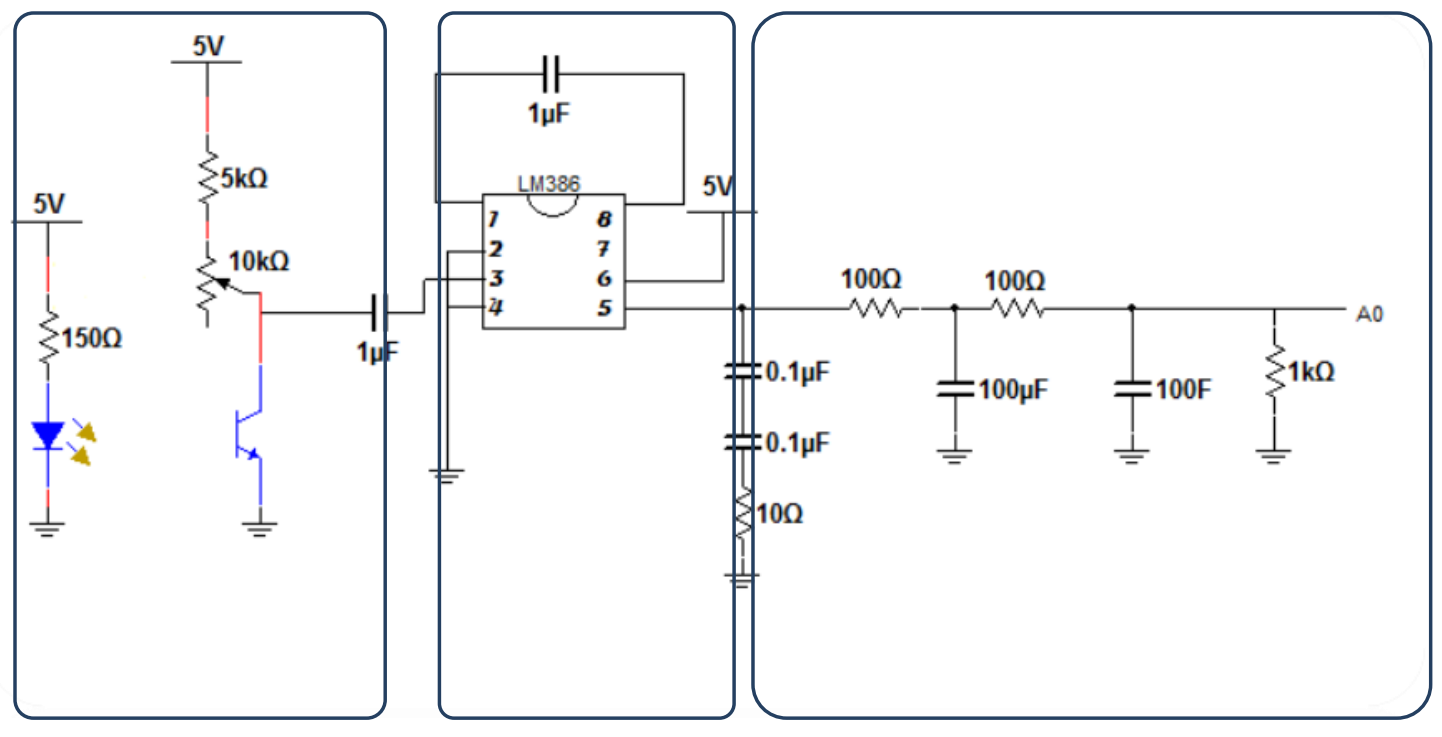

(A) Sensing Process

(B) Amplifying Process

(C) Filtering Process

Figure 3. Processes of the sensing circuit stage

\subsubsection{Stage Two (Filtering and Mapping Stage)}

In this stage, Arduino microcontroller is a platform that performs signal processing received from the sensing signal process in stage one. Arduino received an analog signal from the port (A0) and filtered it from any noise that may disturb the information via implements a digital low pass RLC type of filter. Also, mapping the data and programmed it to supply a heart rate on the desired GUI view see stage two in Figure 2.

\subsubsection{Stage Three (Network Connecting Stage)}

In the third stage, an Arduino board connecting to the internet by using Ethernet shield based on Wiznet (W5100) Ethernet chip via sending a data over the network to a computer-based application as well smartphone-based application that received signals and displayed. There are two platforms used to present the HP values such as computer-based and smartphone-based applications. From computer end, processing software is used as a Graphical User Interface (GUI) to display the result after set IP address of the browser which it depends on the values of time and amplitude with two dimensions (X-axis and Y-axis). Whilst, the result viewed in a smartphone application by using Android Application that programmed by Android Studio software in Java language.

\section{RESULTS AND DISCUSSION}

As for mentioned in the previous section, the primary purpose of this system is transferring the heart pulse in the real-time by using IR emitter sensor, IR detector sensor then sends the heart pulse via Ethernet shield. The result has been shown in the Android and computer applications. For testing the performance of the proposed system, the heart pulse of 10 persons with different (age, gender, and status) are recorded. Table 
1 shows the expected results of the proposed system brespect to the standard heart pulse which taken from

[32]. Figure 4 shows the actual results of ten persons testing with different (age, gender, and status).

Table 1. Expected Results of the Proposed System Testing

\begin{tabular}{cccc}
\hline No. & Gender & Age & Standard [32] \\
\hline 1 & Male & 25 & $95-162$ \\
2 & Male & 23 & $95-162$ \\
3 & Female & 23 & $95-162$ \\
4 & Male & 25 & $95-162$ \\
5 & Female & 51 & $85-145$ \\
6 & Male & 14 & $60-100$ \\
7 & Male & 5 & $75-115$ \\
8 & Female & 70 & $75-128$ \\
9 & Male & 62 & $78-132$ \\
10 & Male & 24 & $95-162$ \\
\hline
\end{tabular}

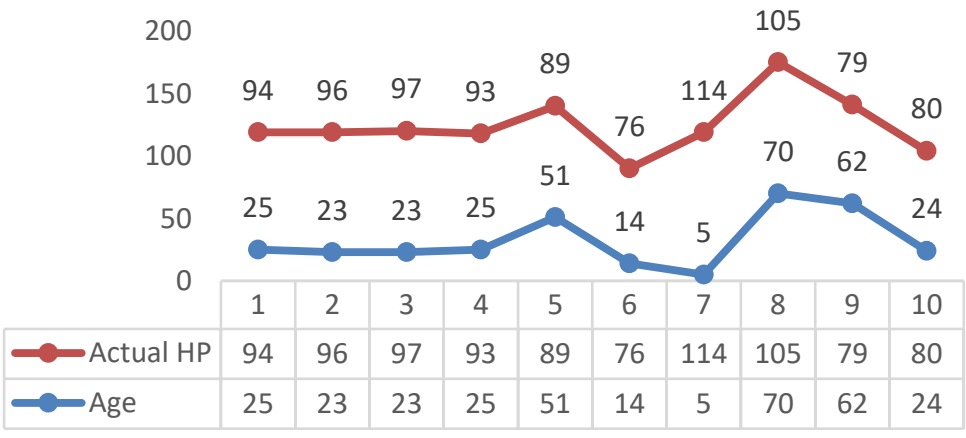

Figure 4. The actual results of ten persons testing with different (age, gender, and status)

The physiological parameters of the human body are usually affected by several factors, such as exercise, body position (for instance, the body position for a short while after standing up quickly), body temperature, and emotions (such as anxiety and arousal). The experiment carries out with a commercial device, and accordingly, we presented an evaluation method based on signal quality and analyzed and tracked the performance of the proposed system in measuring and monitoring the physiological parameters of the patients in real-time settings (see Figure 4). As we cancompared between Table 1 and Figure 4, the HP value recorded by the produced system is lower than the standard HP and this standard value is based on the situation of the patient. Also, the conducted system reported results that all lie within the standard values [32]. The presented healthcare monitoring system was evaluated using the results obtained from 10 persons with different ages, gender, and situations (see Table 1). Additionally, the disccused system measured a patient's physiological parameters, namely, HP. The results showed that the proposed system has potential to be implemented as a decision support system in the healthcare field for future research.

\section{COMPARISON OF THE PREVIOUS HEART RATE MONITORING SYSTEMS IN HEALTHCARE SERVICES AND APPLICATIONS}

Several efforts have been made by researchers on proposing and developing monitoring healthcare systems based on sensor technology to present sustainable medical interventions concerning different metrics such as pure, low energy consumption and real-time feedback. These systems have been introduced to improve the quality of health services and to reduce the total cost of healthcare [33]. Table 2 presents a comparative analysis of previous studies and the proposed system in heart rate monitoring systems for schemes and methods, heart rate parameters, real-time or remote monitoring conditions, and highlights to their main findings. 
Table 2. Comparison of the Previous Studies for Heart Rate Monitoring Systems

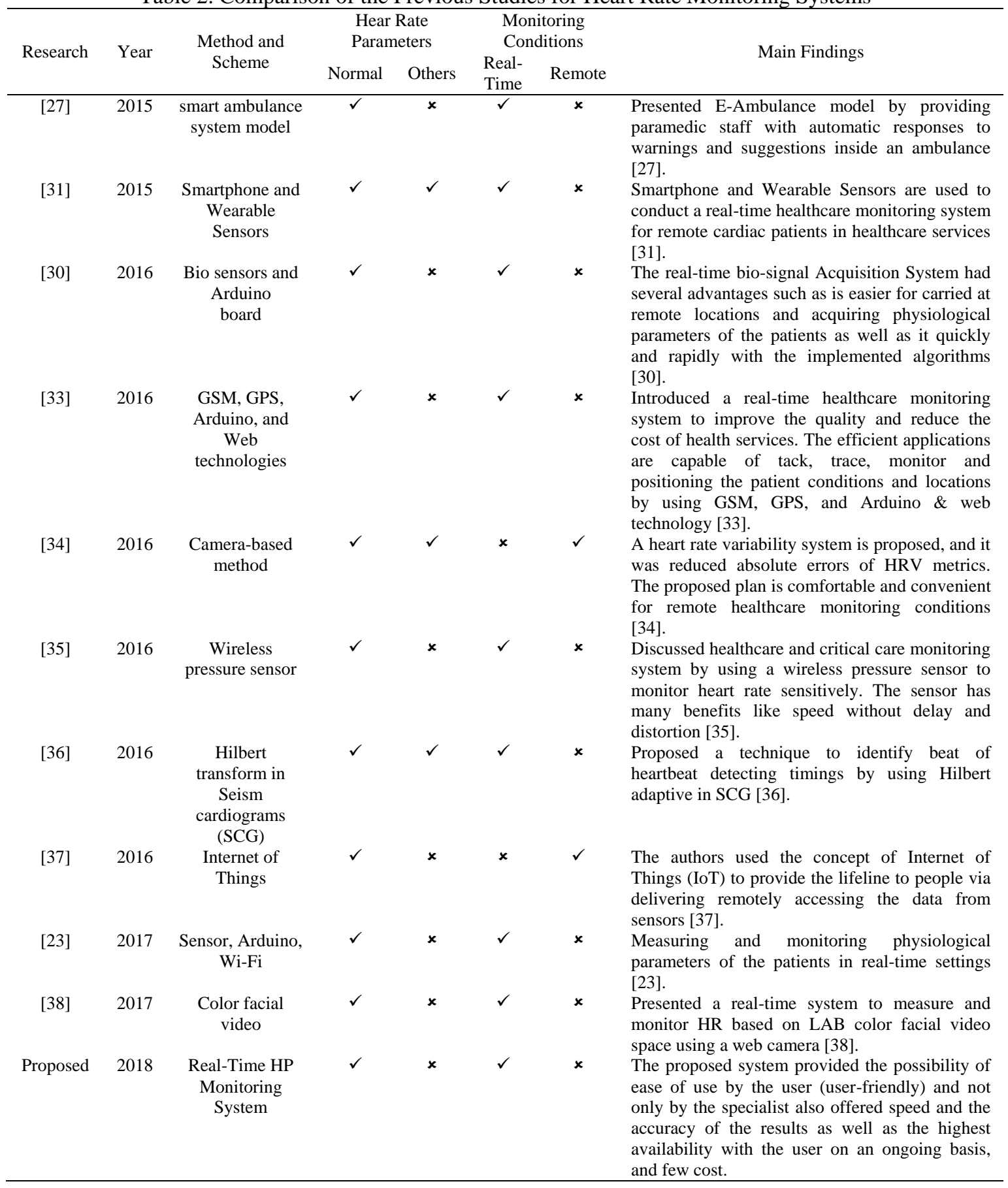

Most previous studies that measured the heart rate of patients in a real-time environment such as [23], [27], [30], [31], [33], [35], [36], and [38]. Whilst, two studies are measured HR remotely by [34] and [37].

On the other hands, regarding heart rate parameters. There are wireless, wired, Bluetooth, internetbased, and webcam communication. Seven previous studies conducted a heart rate in the normal condition like [23], [27], [30], [33], [35], [37], and [38]. Whereas, the works [31], [34], and [36] are presented in several heart rate parameters such as heartbeat timings and inter-beat time intervals in SCG by [36], heart rate variability by [34], Normal HR, Bradycardia HR, and Tachycardia HR by [31]. 


\section{CONCLUSIONS AND FUTURE DIRECTIONS}

Monitoring systems for hospital healthcare are necessary to continue monitoring and tracking the physiological parameters for the patient. Advanced applications have been presented via wireless technologies to bring forth exciting possibilities in the medical market. Today, wireless technology has been eliminated all of the time-consuming jobs as well as, the patients could be liberated from instrumentation and bed. In this paper, design a system for measuring heart pulse of a patient and displaying it in a smartphone and computer over the network in real-time settings. The presented method provided the possibility of ease of use by the user and not only by the specialist also offered speed and the accuracy of the results as well as the highest availability with the user on an ongoing basis, and few cost. From the perspective of future work, we intend to develop the conducted system to encompass two directions, first direction is to improve the proposed system to measure heart rate, temperature, Oxygen in the blood and breathing. The second direction target is to enhance the application that performs tracking to the patient physiological parameter by a doctor via sending alarm or notification. In addition to, send an alert to a nearby clinical home via GPS for an emergency situation.

\section{REFERENCES}

[1] Ebrahim et al, "Epidemiology in old age/ edited by Shah Ebrahim and Alex Kalache", London: BMJ Publishing Group. pp. 22-31, 1996. Avaiable online url: http://www.who.int/iris/handle/10665/41921.

[2] Gray JM. Better value healthcare-the 21st century agenda. Zeitschrift für ärztliche Fortbildung und Qualität im Gesundheitswesen-German Journal for Quality in Health Care. 2007 Jun 29; 101(5): 344-6.

[3] Ogunduyile OO, Olugbara OO, Lall M. Development of wearable systems for ubiquitous healthcare service provisioning. APCBEE procedia. 2013 Jan 1; 7: 163-8.

[4] Hao Y, Foster R. "Wireless body sensor networks for health-monitoring applications". Physiological measurement. 2008 Oct 9; 29(11): R27.

[5] Pallin DJ, Espinola JA, Camargo CA. "US population aging and demand for inpatient services". Journal of hospital medicine. 2014 Mar 1; 9(3): 193-6.

[6] Baig MM, Gholamhosseini H. "Smart health monitoring systems: an overview of design and modeling". Journal of medical systems. 2013 Apr 1; 37(2): 9898.

[7] Minaie A, Sanati-Mehrizy A, Sanati-Mehrizy P, Sanati-Mehrizy R. "Application of wireless sensor networks in health care system". age. 2013; 23(1).

[8] Kit CY, Tomari R, Zakaria WN, Othman N, Safuan SN, Yi JA, Sheng NT. "Mobile based Automated Complete Blood Count (Auto-CBC) Analysis System from Blood Smeared Image". International Journal of Electrical and Computer Engineering (IJECE). 2017 Dec 1; 7(6): 3020-9.

[9] Dorr D, Bonner LM, Cohen AN, Shoai RS, Perrin R, Chaney E, Young AS. "Informatics systems to promote improved care for chronic illness: a literature review". Journal of the American Medical Informatics Association. 2007 Mar 1; 14(2): 156-63.

[10] Caldeira JM, Rodrigues JJ, Lorenz P. "Toward ubiquitous mobility solutions for body sensor networks on healthcare". IEEE Communications Magazine. 2012 May; 50(5): 108-15.

[11] Pantelopoulos A, Bourbakis NG. "A survey on wearable sensor-based systems for health monitoring and prognosis". IEEE Transactions on Systems, Man, and Cybernetics, Part C (Applications and Reviews). 2010 Jan; 40(1): $1-2$.

[12] Ibrahim N, Tomari R, Zakaria WN, Othman N. "Non-contact Heart Rate Monitoring Analysis from Various Distances with different Face Regions". International Journal of Electrical and Computer Engineering (IJECE). 2017 Dec 1; 7(6): 3030-6.

[13] Magrabi F, Ong MS, Runciman W, Coiera E. "An analysis of computer-related patient safety incidents to inform the development of a classification". Journal of the American Medical Informatics Association. 2010 Nov 1; 17(6): 663-70.

[14] Li S, Ding X, Yang T. "Analysis of five typical localization algorithms for wireless sensor networks". Wireless Sensor Network. 2015 Apr 30; 7(04): 27.

[15] Magrabi F, Aarts J, Nohr C, Baker M, Harrison S, Pelayo S, Talmon J, Sittig DF, Coiera E. "A comparative review of patient safety initiatives for national health information technology". International journal of medical informatics. 2013 May 1; 82(5): e139-48.

[16] Rjeib HD, Ali NS, Al Farawn A, Al-Sadawi B, Alsharqi H. "Attendance and Information System using RFID and Web-Based Application for Academic Sector". International Journal of Advanced Computer Science and Applications. 2018; 9(1).

[17] Wang D, Wang P. "Understanding security failures of two-factor authentication schemes for real-time applications in hierarchical wireless sensor networks". Ad Hoc Networks. 2014 Sep 1; 20: 1-5.

[18] Virone G, Wood A, Selavo L, Cao Q, Fang L, Doan T, He Z, Stankovic J. "An advanced wireless sensor network for health monitoring". InTransdisciplinary conference on distributed diagnosis and home healthcare (D2H2) 2006 Apr 2 (pp. 2-4). 
[19] Callen J, Hordern A, Gibson K, Li L, Hains IM, Westbrook JI. "Can technology change the work of nurses? Evaluation of a drug monitoring system for ambulatory chronic disease patients". International journal of medical informatics. 2013 Mar 1; 82(3): 159-67.

[20] Wiharto W, Kusnanto H, Herianto H. "Hybrid system of tiered multivariate analysis and artificial neural network for coronary heart disease diagnosis". International Journal of Electrical and Computer Engineering (IJECE). 2017 Apr 1; 7(2): 1023-31.

[21] Jovanov E, Milenkovic A. "Body area networks for ubiquitous healthcare applications: opportunities and challenges". Journal of medical systems. 2011 Oct 1; 35(5): 1245-54.

[22] Jafari R, Encarnacao A, Zahoory A, Dabiri F, Noshadi H, Sarrafzadeh M. "Wireless sensor networks for health monitoring". In Mobile and Ubiquitous Systems: Networking and Services, 2005. MobiQuitous 2005. The Second Annual International Conference on 2005 Jul 17 (pp. 479-481). IEEE.

[23] Ali NS, Alyasseri ZA. "Wireless Sensor Network and Web Application Hybrid Scheme for Healthcare Monitoring". Journal of Soft Computing and Decision Support Systems. 2017 Aug 19; 4(5): 1-7.

[24] Aminian M, Naji HR. "A hospital healthcare monitoring system using wireless sensor networks". J. Health Med. Inform. 2013 Feb; 4(02): 121.

[25] González FC, Villegas OO, Ramírez DE, Sánchez VG, Domínguez HO. Smart multi-level tool for remote patient monitoring based on a wireless sensor network and mobile augmented reality. Sensors. 2014 Sep 16; 14(9): 1721234.

[26] Triantafyllidis A, Velardo C, Shah SA, Tarassenko L, Chantler T, Paton C, Rahimi K. "Supporting heart failure patients through personalized mobile health monitoring". In Wireless Mobile Communication and Healthcare (Mobihealth), 2014 EAI 4th International Conference on 2014 Nov 3 (pp. 287-290). IEEE.

[27] Almadani B, Bin-Yahya M, Shakshuki EM. "E-AMBULANCE: real-time integration platform for heterogeneous medical telemetry system". Procedia Computer Science. 2015 Jan 1; 63: 400-7.

[28] Mahgoub MT, Khalifa OO, Sidek KA, Khan S. "Health monitoring system using Pulse Oximeter with remote alert". In Computing, Control, Networking, Electronics and Embedded Systems Engineering (ICCNEEE), 2015 International Conference on 2015 Sep 7 (pp. 357-361). IEEE.

[29] Tamura T, Sekine M, Tang Z, Yoshida M, Takeuchi Y, Imai M. "Preliminary study of a new home healthcare monitoring to prevent the recurrence of stroke”. In Engineering in Medicine and Biology Society (EMBC), 2015 37th Annual International Conference of the IEEE 2015 Aug 25 (pp. 5489-5492). IEEE.

[30] Akshay N, Krishna GV. "Design \& implementation of real time bio-signal acquisition system for quality health care services for the population of rural India". In Recent Trends in Electronics, Information \& Communication Technology (RTEICT), IEEE International Conference on 2016 May 20 (pp. 1315-1319). IEEE.

[31] Kakria P, Tripathi NK, Kitipawang P. "A real-time health monitoring system for remote cardiac patients using smartphone and wearable sensors". International journal of telemedicine and applications. 2015 Jan 1;2015:8.

[32] Medical news today, Markus MacGill and Dr Helen Webberley. (2015, September 10), Retrieved From http://www.medicalnewstoday.com/articles/235710.php?page=2.

[33] Aziz K, Tarapiah S, Ismail SH, Atalla S. "Smart real-time healthcare monitoring and tracking system using GSM/GPS technologies". In Big Data and Smart City (ICBDSC), 2016 3rd MEC International Conference on 2016 Mar 15 (pp. 1-7). IEEE.

[34] Huang RY, Dung LR. "Measurement of heart rate variability using off-the-shelf smart phones". Biomedical engineering online. $2016 \mathrm{Jan} ; 15(1): 11$.

[35] Shin KY, Lee JS, Jang J. "Highly sensitive, wearable and wireless pressure sensor using free-standing ZnO nanoneedle/PVDF hybrid thin film for heart rate monitoring". Nano Energy. 2016 Apr 1; 22:95-104.

[36] Tadi MJ, Lehtonen E, Hurnanen T, Koskinen J, Eriksson J, Pänkäälä M, Teräs M, Koivisto T. "A real-time approach for heart rate monitoring using a Hilbert transform in seismocardiograms". Physiological measurement. 2016 Sep 28; 37(11): 1885 .

[37] Rajput DS, Gour R. "An IoT framework for healthcare monitoring systems". International Journal of Computer Science and Information Security. 2016 May 1; 14(5): 451.

[38] Fernandes SL, Gurupur VP, Sunder NR, Arunkumar N, Kadry S. "A novel nonintrusive decision support approach for heart rate measurement”. Pattern Recognition Letters. 2017 Jul 4. 


\section{BIOGRAPHIES OF AUTHORS}
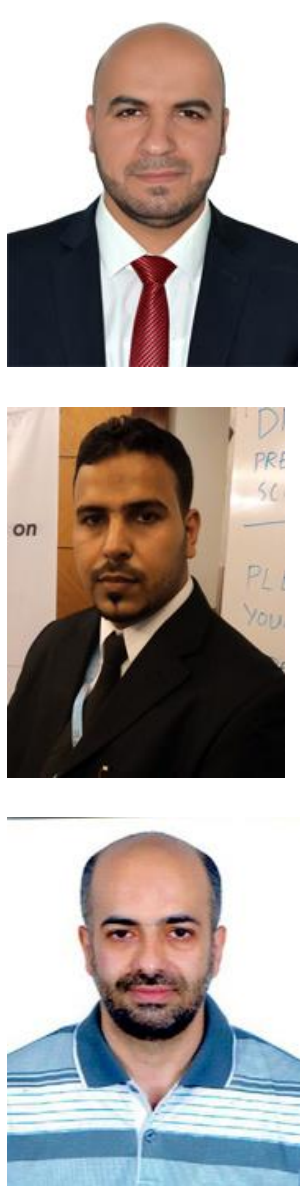

Nabeel Salih Ali received his BSc. degree in computer science from the University of Technology (UoT), Baghdad, Iraq, and the MSc. degree in Computer Science (Internet working Technology) from the University Technical Malaysia Melaka (UTeM), Malaysia, in July 2015. He has been working in Information Technology Research and Development Centre, the University of Kufa from 2007 as a member in addition, as a Lecturer at the Department ofElectronics and communications (ECE), Faculty of Engineering. His research work in web applications security techniques to improve the security and survivability of computer systems. In addition to, Wireless Sensor Network (WSN), healthcare monitoring systems, Internet of Things (IoT), password encryption, andSoftware Defined Network (SDN).

Zaid Abdi Alkareem Alyasseri: (b. 1985 Najaf/Iraq) graduated with a BSc in computer science from Babylon University in 2007 and received MSc in computer science from University Science Malaysia (USM) in 2013. The author works as an Asst. Lecturer in Electronics and Communication Engineering Dept. at the University of Kufa-Iraq. He has experience for 10 years in software development and system analysis also he has goodexperience in many programming languages and database. Authors' research interests are computer vision and image processing, computer graphics processing, programming, and parallel computing.

Abdulhussein Abdulmohson, he was born in Al-Najaf, Iraq in 1979. He obtained the B. Tech. Degree in Computer Engineering from University of Technology, Baghdad, Iraq, 2002. M.Tech. Degree in computer science and engineering from Osmania University, Hyderabad, India Hyderabad, India. He is a lecturer in the Faculty of education at the University of Kufa, Najaf, Iraq where he has been a faculty member since 2015 . 